\title{
Psychopathologies and socioeconomic status as risk indicators for periodontitis: a survey-based investigation in German dental practices
}

\author{
Maria Lenk ${ }^{1} \cdot$ Barbara Noack $^{2} \cdot$ Kerstin Weidner $^{1} \cdot$ Katrin Lorenz $^{2}$ (D)
}

Received: 6 August 2021 / Accepted: 26 October 2021 / Published online: 8 November 2021

(c) The Author(s) 2021, corrected publication 2022

\begin{abstract}
Objectives Periodontitis is a highly prevalent multifactorial disease associated with various mental disorders. However, study results about this association are still contradictory. One methodological reason could be the neglect of potential confounders, such as socioeconomic factors or mental comorbidity. Our study examined a wide range of potential psychosocial risk indicators to identify those with relevant associations to periodontitis.

Materials and methods In a cross-sectional study, 111 patients with periodontitis (PERIO) ( $>30 \%$ teeth with approximal attachment loss $\geq 5 \mathrm{~mm}$ ) and 110 patients without periodontitis (NON-PERIO) were recruited in four dental practices in Germany. Clinical attachment loss, pocket depth, plaque, bleeding on probing, and DMFT were measured. Psychopathologic symptoms and socioeconomic status were recorded using self-report questionnaires (DAS, PHQ-8, GAD-7, CTS, SCOFF, AUDIT, FTND, SSS-8, SES).

Results The PERIO group reported significantly lower socioeconomic status (Cohen's $d=0.49$ ) and higher psychopathological symptom burden than the NON-PERIO regarding dental anxiety $(d=0.86)$ and avoidance behavior, nicotine dependency $(d=0.84)$, depressiveness $(d=0.46)$, general anxiety $(d=0.45)$, somatic symptoms $(d=0.42)$, and childhood traumatization $(d=0.34)$. No significant group differences existed for alcohol abuse and eating disorders. Dental anxiety was the strongest predictor of periodontitis and showed significant correlations with other psychopathologies and social status.

Conclusions Out of all psychosocial factors, socioeconomic status and dental anxiety showed the greatest association with periodontitis.

Clinical relevance Dentists should encourage socially disadvantaged and dentally anxious patients in the utilization of prevention and dental care. Furthermore, physicians and psychotherapists can contribute to the early detection of dental anxiety, oral diseases, and avoidance behavior.
\end{abstract}

Keywords Periodontitis $\cdot$ Dental anxiety $\cdot$ Socioeconomic status $\cdot$ Mental disorders

\section{Introduction}

Periodontitis is a highly prevalent multifactorial inflammatory disease associated with dysbiotic plaque biofilm, as recently stated at the World Workshop in 2017 [1].

Katrin Lorenz

katrin.lorenz@tu-dresden.de

1 Department of Psychotherapy and Psychosomatics, Carl Gustav Carus Faculty of Medicine, Technische Universität Dresden, Fetscherstr. 74, 01307 Dresden, Germany

2 Department of Periodontology, Technische Universität Dresden, Fetscherstr, 74, 01307 Dresden, Germany
According to the concept of pathogenesis by Page and Kornman [2], acquired factors like smoking, uncontrolled diabetes, and genetic disposition can modify the immuneinflammatory response of the host and/or the soft and hard tissue metabolism. Up to now, smoking and diabetes are the only risk factors included as periodontitis modifiers in the newly proposed staging scheme [3], while emotional stress and depression are classified as systemic disorders that may contribute to attachment loss [4].

During the past 20 years, the role of psychopathological factors gained attention in periodontitis research. Associations between periodontitis and depression [5], anxiety disorders [6], alcohol consumption and dependence [7], and post-traumatic stress disorder [8] were discussed. However, 
most studies only investigated the influence of one psychopathology on periodontitis, neglecting important interactions due to psychological comorbidities and social class dependence. These complex interrelations may be one of the reasons why study results are sometimes contradictory. As an example, the association between depression and periodontitis was confirmed in some studies but in others not [9]. For both prevention and therapy of periodontitis, it is of utmost interest to investigate psychosocial factors taking into account their mutual link. The biopsychosocial model of health [10] considers biological, psychological, and social interacting factors as determinants. Within this model, the socioeconomic status plays an important role for either health or disease. Impaired working and living conditions, low education, or low income can provoke pathobiological conditions [11]. Low socioeconomic status can cause chronic stress in an individual, and therefore, biological mechanisms like an activation of the hypothalamic-pituitary-adrenal axis are initiated. Resulting permanent high cortisol levels and a pro-inflammatory immune status can affect the susceptibility to periodontal inflammation [12]. Moreover, dental anxiety is a highly prevalent and important psychological risk factor for oral health impairment [13, 14]. A German survey revealed low levels of dental anxiety in $60 \%$, moderate levels in $23 \%$, and high levels of dental anxiety in $17 \%$ of the population studied. Dental phobia was recognized in $11 \%$ [15]. Dental anxiety is a common comorbidity of various mental disorders [16]. In own observations, about $42 \%$ of patients with post-traumatic stress disorder, $31 \%$ with anxiety disorders, and $21 \%$ with depressive disorders presented a high degree of dental fear [17]. However, this relationship is seldom recognized and needs further attention within the model of pathogenesis of periodontitis.

Smoking is the only environmental factor that is recognized as true risk factor for periodontitis in the pathogenesis model [2]. It affects the progression rate (higher severity and extent of the disease at an earlier age) as well as the treatment response (less improvement in attachment level and inflammation, higher rates of tooth loss during maintenance care) $[3,18]$. DNA damage, a chronic inflammatory state, and oxidative stress provoked by tobacco components are responsible for local and systemic alterations in smokers. Smoking is more prevalent in low social class and in persons suffering from mental illnesses [19, 20]. About twice as many patients with mental disorders smoke compared to the general population [21-23]. At the same time, there is an increased risk in smokers for alcohol-related diagnoses [24], again highlighting the complex interactions between several environmental factors and mental diseases or symptoms.

While still an unclear association between alcohol consumption and periodontitis exists when single studies are considered, a meta-analysis including more than 90,000 participants from 18 studies reported a relative risk for periodontitis of 1.6. A sub-analysis of six studies revealed a linear dose-response relationship between alcohol intake and periodontitis [25]. Only 10 of the 18 single studies included socioeconomic conditions like education and income as covariates. Adjustments for smoking were done in 15 studies. However, comorbidities like depression or dental anxiety were never considered.

The aim of this study was to examine a wide range of psychopathological and socioeconomic factors to identify those primarily associated with periodontitis.

The following hypotheses were studied:

- Periodontitis and non-periodontitis patients differ regarding psychopathological symptom burden.

- Periodontitis and non-periodontitis patients differ regarding socioeconomic status.

- Psychopathological symptoms, social status, and dental parameters are intercorrelated.

- The joint consideration of potential psychosocial risk indicators enables the selection of those factors, which are associated with periodontitis to a relevant extent.

\section{Methods}

In a cross-sectional study, 111 periodontitis patients (periodontitis group) and 110 patients without periodontitis (control group) were consecutively recruited in four urban private dental practices in Germany (Aalen, Berlin, Esslingen, Rottweil). The investigating dentists were trained to apply periodontal examination techniques. The training included oral and written instructions and practice on index assessment and periodontal probing under supervision of a senior clinician. The study took place from January 2015 to February 2016. Written informed consent was obtained from every participant before inclusion. The study was approved by the Ethical Review Board of the Technische Universität Dresden (EK 361092014).

\section{Participants}

Men and women $\geq 40$ years of age who gave informed consent and either met criteria for PERIO or NON-PERIO groups were included. All participants received a periodontal examination including probing pocket depths (PD) and clinical attachment loss (CAL). Patients who presented more than $30 \%$ teeth with approximal CAL $\geq 5 \mathrm{~mm}$ were assigned to the PERIO group [26]. Therefore, all patients were diagnosed with generalized periodontitis stage III or IV as defined in the current classification [3]. Patients who had not more than one tooth with $\mathrm{CAL} \geq 4 \mathrm{~mm}$ or probing depths $\geq 5 \mathrm{~mm}$ qualified for the NON-PERIO group.

Exclusion criteria were: 
Less than 15 teeth

Severe systemic diseases (e.g., infectious diseases, uncontrolled diabetes)

Current radiation or chemotherapy

Prescription of anticoagulants

Requirement of antibiotic treatment for dental appointments

Medication intake that affects the gingival condition

Pregnancy or breastfeeding

Unability to assess the nature and consequences of study participation

\section{Parameters}

All participants received a dental examination including documentation of decayed, missing, and filled teeth (DMFT). The index represents the sum of diseased teeth in relation to the possible number of teeth $(n=28)$ in a dentition. PD, CAL, bleeding on probing (BOP), plaque index (PI) [27], and gingival index (GI) [28] were documented at six sites per tooth.

In addition, the following questionnaires (all versions in German) to assess psychopathologies, nicotine and alcohol abuse, and socioeconomic burden were used:

- Dental Anxiety Scale (DAS) [29]: four-item questionnaire measuring dental anxiety. Range 4-20. Individuals with a score $\geq 15$ were classified as high dental anxious. In addition to the DAS, anxiety-related avoidance behavior was recorded, whereby cancellations/non-attendance of dental appointments and the period of avoiding dental appointments in years were queried.

- Patient Health Questionnaire Depression Scale (PHQ-8) [30]: derived from the depression module of the Patient Health Questionnaire (PHQ-D), the PHQ-8 measures depressiveness with eight items. Range 0-24.

- General Anxiety Disorder (GAD-7) [31] is a 7-item module of the PHQ-D for measuring symptom severity of generalized anxiety. Range $0-21$.

- Somatic Symptom Scale (SSS-8) [32] is an 8-item brief version of the PHQ module for accessing perceived burden of common somatic symptoms. Range 0-32.

- Childhood Trauma Screener (CTS) [33] is the short form of the Childhood Trauma Questionnaire for screening recalled abuse and neglect in childhood and youth by five items. Range 5-25.

- Eating disorder (SCOFF) [34] is a screening questionnaire for anorexia nervosa and bulimia nervosa consisting of five dichotomous items. Range 0-5.

- Alcohol Use Disorders Identification Test (AUDIT) [35] is a questionnaire developed on behalf of the WHO to screen for harmful alcohol consumption. With 10 items, alcohol consumption but also drinking behavior and alcohol-related problems are recorded. Range 0-40.

- Fagerström test for nicotine dependence (FTND) [36]: the first item is used to distinguish between smokers, former smokers, and non-smokers. Only current smokers complete six additional questions to capture criteria for nicotine dependence. Based on the total FTND score (range 0 to 10), a categorization into five severity categories can be made. Note, in our regression analysis, we used a modified variable (called FTND*) that includes smoking and currently non-smoking participants (FTND* variable coding: $1=$ non-smoker; $2=$ former smoker; 3 = severity category, none/very low nicotine dependency (FTND 0-2); $4=$ low nicotine dependency (FTND 3-4); $5=$ medium nicotine dependency (FTND 5); $6=$ strong nicotine dependency (FTND 6-7); $7=$ very strong nicotine dependency (FTND 8-10)).

- Socioeconomic Status Index (SES) [31] is a multidimensional index that captures socioeconomic status in the three domains (subscales) income, education, and profession. Score values are assigned to the response categories so that each subscale takes on a quasi-metric value ranging from 1.0 to 7.0 points. The sum of the three scales results in a total score representing socioeconomic status (range 3-21). The higher the sum score, the higher the social status. The scale education reflects the individually acquired schooling and professional degree. For the scale profession status, the current occupation held by the participant and the main earner in the household is recorded. The income scale is derived from household net income weighted by the age-appropriate financial needs of household residents.

The time to answer the questionnaires averaged $30 \mathrm{~min}$. For further information on questionnaire design, validity, and reliability, please refer to the primary literature provided.

\section{Statistical analysis}

Prior to the study, we estimated the minimum sample size required to detect at least medium effect sizes. Medium effects were defined as sufficient, as we aimed to identify differences relevant for clinical practice. To ensure the detection of medium effects $(d=0.5)$ when comparing two equally large, independent groups with a significance level of $5 \%$ and a power $(1-\beta)$ of $0.8,100$ persons per group had to be recruited plus $10 \%$ more in case of drop-outs or missing values [37].

Statistical analyses were carried out using SPSS 27 (IBM Corp., Armonk, NY, USA). Missing values were not imputed. Deviations from total $N$ within single scales were reported. Medians $[\tilde{\mathrm{x}}]$ and quartiles $\left[x_{.25}, x_{.75}\right]$ were shown as descriptive values. Since some of the variables were not 
normal distributed, non-parametric statistical tests were performed. Group differences of categorical variables were examined using Fisher's exact tests. For ordinal or metric variables Mann-Whitney $U$ tests were used, and in addition, Cohen's $d$ was reported as a measure for effect strength. Correlative associations were analyzed using Spearman correlations.

After comparing PERIO and NON-PERIO groups regarding psychological symptom burden and SES, all variables which showed inter-group differences were included in logistic regression analyses (method, forward stepwise likelihood ratio). The regression model was adjusted for age because PERIO patients were significantly older than NONPERIO patients were.

\section{Results}

\section{Demographic characteristics}

In the PERIO group, 111 participants finished the study. The NON-PERIO group consisted of 110 participants without periodontitis. In both groups, more women were included, but gender distribution did not differ significantly between both groups (Fisher's exact test, $p=0.056$; NON-PERIO female, $65.5 \%$ ( $n=72)$; PERIO female, 52.3\% $(n=58)$ ). Participants in the PERIO group were significantly older then participants from the NON-PERIO group ( $U$ test, $\mathrm{z}=4.101$; $\mathrm{p}<0.001 ;$ NON-PERIO, $\tilde{\mathrm{x}}=47.0$ years, $\mathrm{x}_{.25}=42.0$ years, $\mathrm{x}_{.75}=56.0$ years; PERIO, $\tilde{\mathrm{x}}=54.0$ years, $\mathrm{x}_{.25}=46.0$ years, $\mathrm{x}_{.75}=63.0$ years).

\section{Clinical parameters}

As expected, periodontitis patients had significant higher PD and CAL, more inflammation (BOP, GI) and plaque (PI), and presented less teeth and higher DMFT scores than NONPERIO group participants (Table 1).

\section{Socioeconomic status}

Socioeconomic status was significantly lower in the PERIO group compared to the NON-PERIO group (SES $\left.d_{\text {cohens }}=-0.49\right)$. These differences reached statistical significance in all three status domains (income $d_{\text {cohens }}=-0.30$, profession $d_{\text {cohens }}=-0.37$ ), with the strongest effects for the group differences in the domain education $\left(d_{\text {cohens }}=-0.55\right)$. SES and its subdomains showed significant negative correlations with the plaque index, gingival index, and CAL. The lower SES, the higher were the index values and attachment loss. However, no significant correlation between SES and DMFT index could be detected (Table 1). Psychopathological symptoms were negatively correlated with
SES (all correlations $p<0.05$; DAS, $r_{\mathrm{s}}=-0.233$; GAD$7, r_{\mathrm{s}}=-0.147$; PHQ-8, $r_{\mathrm{s}}=-0.238 ;$ CTS, $r_{\mathrm{s}}=-0.295$; FTND, $\left.r_{\mathrm{s}}=-0.304\right)$.

\section{Psychopathological symptom severity}

Periodontitis patients reported a higher intensity of psychopathologic symptoms than participants without periodontitis did. The PERIO group reported significant higher dental anxiety (DAS, $d_{\text {cohens }}=0.86$ ), nicotine dependence in smokers (FTND, $d_{\text {cohens }}=0.84$ ), depression (PHQ-8, $d_{\text {cohens }}=0.46$ ), symptoms of general anxiety $\left(\mathrm{GAD}-7, d_{\text {cohens }}=0.45\right)$, somatic symptom burden (SSS$8, d_{\text {cohens }}=0.42$ ), and childhood traumatization (CTS, $d_{\text {cohens }}=0.34$ ) than the NON-PERIO group (Table 1 ). No statistically significant group differences were found concerning symptoms of eating disorders (SCOFF) or alcohol dependency (AUDIT).

Out of all psychological symptom burden variables, the strongest effect could be observed for dental anxiety. A total of $18.9 \%(n=21)$ of patients in the PERIO group suffered from severe dental anxiety compared to only $3.6 \%(n=4)$ in the NON-PERIO group. Moderate dental anxiety was found in $11.7 \%(n=13)$ of the PERIO participants versus $3.6 \%(n=4)$ of NON-PERIO participants, while mild or no anxiety was reported in $69.4 \%(n=77)$ PERIO participants compared to $92.7 \%(n=102)$ NON-PERIO participants.

Dental anxiety-related avoidance behavior was reported significantly more frequently by PERIO patients. Due to dental fear, $24.5 \%(n=27)$ periodontitis patients had already cancelled or have not attended dental appointments compared to $7.3 \%(n=8)$ NON-PERIO participants (Fisher's exact test, $n=219 ; p<0.001$ ). Furthermore, $30.9 \%(n=34)$ periodontitis patients and $6.4 \%(n=7)$ NON-PERIO participants avoided dental appointments for more than 1 year (Fisher's exact test, $n=219 ; p<0.001$ ). Among these patients with anxiety-related avoidance behavior, the median of the longest continuous period of avoiding dental visits was 3 years in the PERIO group $\left(\tilde{\mathrm{x}}=3\right.$ years, $x_{.25}=2$ years, $\mathrm{x}_{.75}=5$ years, maximum $=18$ years $)$ and 2 years in the NON-PERIO group ( $x=2$ years, $\mathrm{x}_{.25}=$ 1,5 years, $x_{.75}=6$ years, maximum $=10$ years).

Regarding the number of smokers, the two groups did not differ significantly (Fisher's exact test $: n=221 ; p=0.303$ ). Based on patients' self-reports, $27.9 \%(n=31)$ of PERIO patients versus $20.9 \%(n=23)$ of the NON-PERIO participants were current smokers. Furthermore, 37.8\% $(n=42)$ versus $35.5 \%(n=39)$ were former smokers and $34 \%(n=38)$ versus $43.6 \%(n=48)$ were non-smokers, respectively. However, the groups differed strongly in the severity of nicotine dependence. Periodontitis patients who smoked had a higher nicotine dependence 


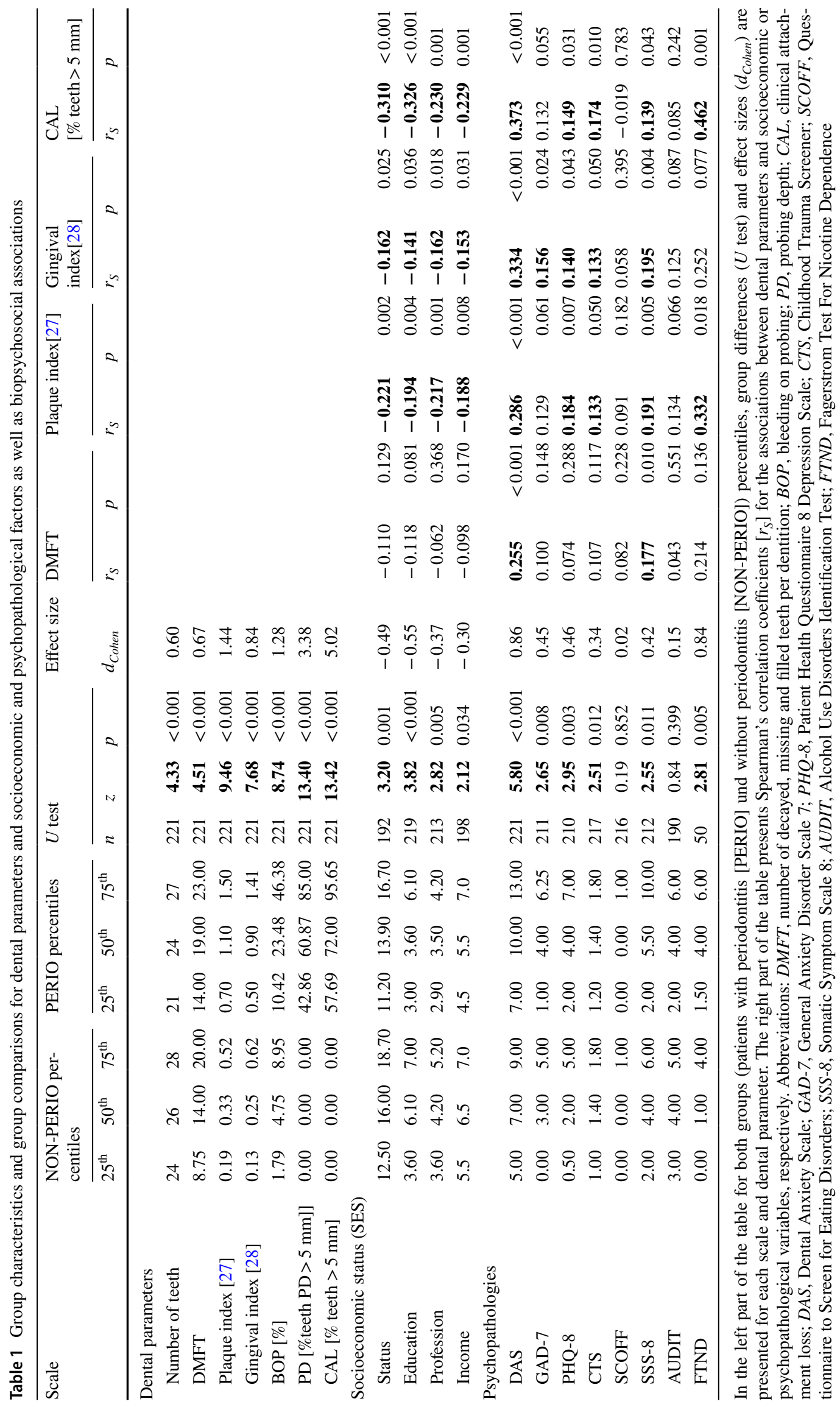


than smokers of the NON-PERIO group (FTND, Utest : $n=50 ; z=2.81 ; p=0.005 ; d=0.84$ ).

All variables of psychological symptom burden, which showed significant group differences, significantly correlated with PI, GI, or attachment loss. DMFT index was only correlated with dental anxiety (DAS) and somatic symptom burden (SSS-8) (compare Spearman rank coefficients in Table 1). In addition, intercorrelations existed between psychopathological symptoms. For example, dental anxiety was correlated $(p<0.01)$ with symptoms like depressiveness (PHQ-8, $\left.r_{S}=0.361 ; n=210\right)$, general anxiety $\left(\mathrm{GAD}-7, r_{S}=0.357\right.$; $n=211)$, somatic symptoms (SSS-8, $r_{S}=0.360 ; n=212$ ), and childhood traumatization (CTS, $r_{S}=0.164 ; n=217$ ).

\section{Socioeconomic status und psychopathological symptom burden as predictors for periodontitis}

A logistic regression analysis was performed to extract variables with the greatest predictive impact on periodontitis (dependent variable, group) out of all potential influencing factors (independent variables, age, SES, DAS, FTND* in smokers, PHQ-8, GAD-7, SSS-8, CTS). Dental anxiety (DAS) could be identified as the strongest predictor in the model. After inclusion of dental anxiety, also age, smoking/ nicotine dependence (FTND* in smokers), and socioeconomic status (SES) showed a significant but subordinated influence. All other independent (PHQ-8, GAD-7, SSS8 , CTS) variables had no additional impact and were not included in the model (Table 2).

\section{Discussion}

There is growing evidence that associations between mental disorders and periodontal health exist. This relationship was supported by our current study results that showed a higher psychopathological symptom burden in periodontitis patients compared to periodontal healthy participants. More specifically, significant group differences were detected concerning depression, general anxiety, dental anxiety, childhood traumatization, and nicotine dependence in smokers.

The relationship between each of the mental disorders and periodontal health has been studied to a varying degree. Study results are contradictory and in parts not consistent and comparable. Concerning depression and periodontitis, one meta-analysis did not support associations between the two conditions and showed high heterogeneity between the studies [5]. Araujo et al. could include only seven crosssectional studies but criticized statistical, methodological, and clinical heterogeneity. Studies lacked standardized study designs, disease definitions for both periodontitis and depression, and inclusion criteria for participants and outcome variables. Another meta-analysis of 14 studies found an association but explicitly pointed out to interpret these results with care due to a high heterogeneity [38].

The higher rate of self-reported childhood abuse and neglect we found in PERIO patients $(d=0.34)$ has not been investigated so far by the knowledge of the authors. However, this relationship seems obvious, as abuse, especially sexual abuse, predisposes for dental anxiety and avoidance of dental treatment $[39,40]$. Our results suggest that the link between childhood trauma and periodontitis may be mediated by dental anxiety, as CTS scores had no significant impact any more when dental anxiety was included in the regression model. The only study that addressed the influence of trauma on periodontal health investigated posttraumatic stress disorders in war veterans [8] and is therefore not comparable in terms of content. In contrast, smoking and nicotine dependency are undisputedly recognized environmental risk factors and modifiers of periodontitis progression [3]. Our results have proven this fact by confirming a
Table 2 Results of regression analyses with group (PERIO vs. NON-PERIO) as dependent variable and age, SES, and relevant psychological symptom burden as independent variables

\begin{tabular}{|c|c|c|c|c|c|c|}
\hline \multicolumn{2}{|c|}{ Variables included in the model } & \multirow{2}{*}{$\begin{array}{l}\beta \\
0.233\end{array}$} & \multirow{2}{*}{$\begin{array}{l}p \\
<0.001\end{array}$} & \multirow{2}{*}{$\begin{array}{l}\text { OR } \\
1.262\end{array}$} & \multicolumn{2}{|c|}{$95 \% \mathrm{CI}$ for OR } \\
\hline Step 1 & DAS (dental anxiety) & & & & 1.147 & 1.389 \\
\hline \multirow[t]{2}{*}{ Step 2} & DAS (dental anxiety) & 0.259 & $<0.001$ & 1.295 & 1.170 & 1.434 \\
\hline & Age & 0.072 & $<0.001$ & 1.075 & 1.036 & 1.115 \\
\hline \multirow[t]{3}{*}{ Step 3} & DAS (dental anxiety) & 0.247 & $<0.001$ & 1.280 & 1.153 & 1.420 \\
\hline & FTND* (smoking) & 0.382 & 0.008 & 1.464 & 1.104 & 1.942 \\
\hline & Age & 0.078 & $<0.001$ & 1.081 & 1.041 & 1.122 \\
\hline \multirow[t]{4}{*}{ Step 4} & DAS (dental anxiety) & 0.230 & $<0.001$ & 1.259 & 1.133 & 1.398 \\
\hline & FTND* (smoking) & 0.348 & 0.019 & 1.416 & 1.060 & 1.892 \\
\hline & SES (status) & -0.114 & 0.021 & 0.892 & 0.809 & 0.983 \\
\hline & Age & 0.081 & $<0.001$ & 1.084 & 1.042 & 1.127 \\
\hline
\end{tabular}

Excluded variables: PHQ-8 (depression), GAD-7 (general anxiety), SSS-8 (somatic symptom burden), CTS (childhood traumatization)

Standardized regression coefficients $\beta$, odds ratios [OR] with 95\% confidence interval [CI], and $p$ values 
higher nicotine dependency in smokers of the periodontitis group.

In addition to the methodological problems critically mentioned by Araujo et al. [5], we also suspect a mis-/overinterpretation of some psychopathological factors due to confounding effects. The investigation of only single psychopathologies ignores the existence of two possible confounders: (i) the comorbidity between mental disorders and (ii) the relation to the socioeconomic status.

(i) There is a high comorbidity between mental diseases [41]. Therefore, psychopathological symptoms cannot be considered as distinct entities. They are more or less correlated with each other. This can be exemplified by the significant correlations of dental anxiety with other symptoms such as childhood traumatization $\left(r_{S}=0.164\right)$, depressiveness $\left(r_{S}=0.361\right)$, or general anxiety $\left(r_{S}=0.357\right)$. It is evident that dental anxiety is often associated with other mental disorders [17, 42]. Patients with depression, anxiety disorder, and post-traumatic stress disorder have a disproportionately high incidence of severe dental fear [17].

(ii) Both mental disorders and periodontitis are more common in the low social class [43,44]. Our results show that several psychopathologic symptoms and social status are significantly correlated with plaque, gingivitis, and clinical attachment loss. Applying the SES index, comparisons of the influence of the three status domains education, profession, and income can be made. We found the largest group differences in education $(d=0.55)$. Lower education appears to carry a greater risk for periodontitis than lower financial background. Possibly, higher levels of education facilitate access to prevention information and practical understanding of health-promoting behavior. Based on large-scale representative epidemiologic studies, factors like education, income, and poverty-income ratio even when adjusted for age and gender were consistently proven as factors to negatively influence periodontitis [43, 45]. Apparently, low socioeconomic status and periodontitis are interrelated via complex biopsychosocial interactions. Both oral healthrelated behavioral factors and pro-inflammatory factors like chronic stress [46], metabolic diseases (e.g., diabetes, obesity [47, 48]), and nicotine dependence [18] are relevant risk factors or indicators for periodontitis which are more frequently observed in low social class.

Only by taking comorbidities and socioeconomic status into account, spurious relationships can be uncovered and the complex nature of biopsychosocial interactions understood. The results of the regression model support this statement. As expected, known risk factors like nicotine dependency, age, and social status showed a significant influence on periodontitis. However, the strongest predictor for periodontitis was dental anxiety. Once these factors were included in the model, the other psychopathological symptoms no longer contributed substantially to the prediction of periodontitis. Although dental anxiety and nicotine dependence have a comparably large effect ( $d=0.86$ vs. 0.84 ), the influence of dental anxiety on periodontitis is still underestimated in research and clinical practice. While associations between caries, dental anxiety, and avoidance behavior were discussed extensively, consequences for periodontal tissues were poorly investigated.

There are a few studies providing evidence for associations between periodontitis and dental anxiety [49-51] and studies which did not confirm associations [52,53]. However, the validity and comparability of the studies are restricted by methodological limitations and differences. For example, non-validated instruments or single-items were used to assess dental anxiety [51,52], periodontitis was documented based on self-reports instead of clinical examinations [50], participants were recruited selectively (e.g., only male soldiers) [53], and the studies differed widely in statistical power and thus in detectable effect sizes.

Similar to our approach, Guentsch and co-workers [49] recruited participants in a German dental practice and assessed dental anxiety using DAS and periodontal status by clinical examination. In accordance with our results, a negative effect of dental anxiety on dental and periodontal health was confirmed. In their study, highly anxious patients (MDAS $\geq 19$ ) compared to lower anxious patients (MDAS < 19) reported more often infrequent dental treatment and had more decayed and fewer filled teeth and more BOP indicating a higher degree of inflammation. Although their group comparison did not reach the significance level for attachment loss, this should not be interpreted per se as a negative result or contradiction to our findings. It might be due to the small number of cases in Guentsch's highly anxious group $(n=20)$. Their reported group means and standard deviations corresponded to a medium effect and suggested a trend towards greater attachment loss in high dental anxious patients.

Considering methodological limitations discussed above, two large-scale Chinese studies strengthen the evidence for dental anxiety associated impairments of periodontal health. A representative cross-sectional study in Hong Kong $(N=1000)$ showed significantly higher CAL in participants with self-reported higher dental anxiety versus low or non-anxious participants [51]. Liu and coworkers [50] found significantly higher dental anxiety (using DAS and DFS) in participants with self-reported periodontal disease compared with those without periodontitis. In a recent preprint report, Liu and co-workers even point to a dental anxiety-reducing effect of periodontitis therapy with scaling and root planing [54]. Of even greater importance would be the evaluation of long-term efficacy of anxiety treatment for periodontal health. Future studies on psychotherapy for dental anxiety should include dental and periodontal parameters as secondary outcomes, 
in addition to anxiety reduction as the main efficiency criterion. As dental anxiety responds very well and quickly to cognitive-behavioral therapy [55], it could be an easily modifiable factor to improve dental care utilization. Even one-session treatments and large group exposure treatment can reduce dental anxiety significantly [56, 57].

For clinical implications, this knowledge of psychosocial risk indicators of periodontitis can sensitize dentists to pay attention to information deficits or fear-related avoidance of prevention and treatment services in socially disadvantaged and dentally anxious patients. Based upon this knowledge, dentists can support affected patients individually. However, dentists cannot reach patients who avoid visiting the dentist. Therefore, it is of particular importance that physicians and psychotherapists are informed about periodontitis and its risk indicators. They can become aware of dental anxiety, oral diseases, and avoidance of regular dental visits and contribute to early detection and referral to specialist care.

\section{Strengths and limitations of the method}

As a cross-sectional survey, our study does not permit causal interpretations. The sample represented the typical clientele of patients in German standard dental practices but was not representative for the population. Our recruitment design entailed a selection bias. It excluded individuals who totally avoid dental visits from participating in the study. Patients with dental phobia are underrepresented in dental offices. Therefore, we possibly even underestimate the influence of dental phobia on periodontitis.

Psychopathological symptom severity was assessed using validated and internationally established questionnaires. As short and easy-to-interpret instruments, they are also practical for clinical routine and frequently used as screening instruments. However, no diagnosis should be made on the basis of questionnaires alone. In the case of retrospective self-reports such as childhood traumatization, the possibility of memory bias must be noted [58].

With the SES index, a validated instrument was used that captures socioeconomic status in a very detailed fashion. It enables to compare and to quantify the status domains and provides an easily manageable variable for statistical analyses due to its metric total score [59]. Nowadays, such multidimensional status indices are preferred in large epidemiological studies. For further periodontitis research, it would be desirable to increase the use of sound methods for capturing the complex construct of social status. Instead of the often self-constructed items and response categories, we recommend the use of validated instruments that allow comparability between studies.

\section{Conclusions}

Out of a broad spectrum of psychopathological symptoms, dental anxiety and low socioeconomic status, especially low education, are associated with periodontitis.

Acknowledgements We would like to thank Prof. Dr. Dr. h.c. Thomas Hoffmann for his continuous support during the conduct of this study and Lena Dietrich, Theresa Kissing, Anna Trojan, and Eva-Maria Weyer for performing the clinical examinations and questionnaire data collection.

Author contribution All authors contributed to the study conception and design. Data analysis was performed by Maria Lenk. The manuscript was written by Maria Lenk and Katrin Lorenz. All authors commented on and approved the final manuscript.

Funding Open Access funding enabled and organized by Projekt DEAL.

\section{Declarations}

Ethics approval All procedures performed involving human participants were in accordance with the ethical standards of the ethics committees of the University of Dresden and with the 1964 Helsinki declaration and its later amendments or comparable ethical standards. The study was approved by the ethics committee of the TU Dresden (EK 361092014).

Consent to participate Informed consent was obtained from all individual participants included in the study.

Conflict of interest The authors declare no competing interests.

Open Access This article is licensed under a Creative Commons Attribution 4.0 International License, which permits use, sharing, adaptation, distribution and reproduction in any medium or format, as long as you give appropriate credit to the original author(s) and the source, provide a link to the Creative Commons licence, and indicate if changes were made. The images or other third party material in this article are included in the article's Creative Commons licence, unless indicated otherwise in a credit line to the material. If material is not included in the article's Creative Commons licence and your intended use is not permitted by statutory regulation or exceeds the permitted use, you will need to obtain permission directly from the copyright holder. To view a copy of this licence, visit http://creativecommons.org/licenses/by/4.0/.

\section{References}

1. Papapanou PN, Sanz M, Buduneli N, Dietrich T, Feres M, Fine DH, Flemmig TF, Garcia R, Giannobile WV, Graziani F, Greenwell H, Herrera D, Kao RT, Kebschull M, Kinane DF, Kirkwood KL, Kocher T, Kornman KS, Kumar PS, Loos BG, Machtei E, Meng H, Mombelli A, Needleman I, Offenbacher S, Seymour GJ, Teles R, Tonetti MS (2018) Periodontitis: consensus report of workgroup 2 of the 2017 World Workshop on the Classification of Periodontal and Peri-Implant Diseases and Conditions. J Periodontol 89(Suppl 1):S173-s182. https://doi.org/10.1002/jper. 17-0721 
2. Page RC (2000) Kornman KS (1997) The pathogenesis of human periodontitis: an introduction. Periodontol 14:9-11

3. Tonetti MS, Greenwell H, Kornman KS (2018) Staging and grading of periodontitis: framework and proposal of a new classification and case definition. J Clin Periodontol 45(Suppl 20):S149-s161. https://doi.org/10.1111/jcpe.12945

4. Albandar JM, Susin C, Hughes FJ (2018) Manifestations of systemic diseases and conditions that affect the periodontal attachment apparatus: case definitions and diagnostic considerations. J Clin Periodontol 45(Suppl 20):S171-s189. https://doi.org/10. 1111/jcpe. 12947

5. Araújo MM, Martins CC, Costa LC, Cota LO, Faria RL, Cunha FA, Costa FO (2016) Association between depression and periodontitis: a systematic review and meta-analysis. J Clin Periodontol 43(3):216-228. https://doi.org/10.1111/jcpe.12510

6. Vettore MV, Leao AT, Monteiro Da Silva AM, Quintanilha RS, Lamarca GA (2003) The relationship of stress and anxiety with chronic periodontitis. J Clin Periodontol 30(5):394-402

7. Amaral Cda S, Vettore MV, Leao A (2009) The relationship of alcohol dependence and alcohol consumption with periodontitis: a systematic review. J Dent 37(9):643-651. https://doi.org/ 10.1016/j.jdent.2009.04.011

8. Muhvić-Urek M, Uhac I, Vuksić-Mihaljević Z, Leović D, Blecić N, Kovac Z (2007) Oral health status in war veterans with posttraumatic stress disorder. J Oral Rehabil 34(1):1-8. https://doi. org/10.1111/j.1365-2842.2006.01674.x

9. Zheng DX, Kang XN, Wang YX, Huang YN, Pang CF, Chen YX, Kuang ZL, Peng Y (2021) Periodontal disease and emotional disorders: a meta-analysis. J Clin Periodontol 48(2):180 204. https://doi.org/10.1111/jcpe. 13395

10. Engel GL (1977) The need for a new medical model: a challenge for biomedicine. Science (New York, NY) 196(4286):129-136. https://doi.org/10.1126/science. 847460

11. Krieger N, Chen JT, Coull BA, Selby JV (2005) Lifetime socioeconomic position and twins' health: an analysis of 308 pairs of United States women twins. PLoS Med 2(7):e162. https://doi. org/10.1371/journal.pmed.0020162

12. Gomaa N, Tenenbaum H, Glogauer M, Quiñonez C (2019) The biology of social adversity applied to oral health. J Dent Res 98(13):1442-1449. https://doi.org/10.1177/0022034519876559

13. Carlsson V, Hakeberg M, Wide Boman U (2015) Associations between dental anxiety, sense of coherence, oral health-related quality of life and health behavior-a national Swedish crosssectional survey. BMC Oral Health 15:100. https://doi.org/10. 1186/s12903-015-0088-5

14. Wennström A, Wide Boman U, Stenman U, Ahlqwist M, Hakeberg M (2013) Oral health, sense of coherence and dental anxiety among middle-aged women. Acta Odontol Scand 71(1):256-262. https://doi.org/10.3109/00016357.2012.671362

15. Enkling N, Marwinski G, Jöhren P (2006) Dental anxiety in a representative sample of residents of a large German city. Clin Oral Invest 10(1):84-91. https://doi.org/10.1007/ s00784-006-0035-6

16. Halonen H, Nissinen J, Lehtiniemi H, Salo T, Riipinen P, Miettunen J (2018) The association between dental anxiety and psychiatric disorders and symptoms: a systematic review. Clin Prac Epidemiol Mental Health CP EMH 14:207-222. https://doi.org/ 10.2174/1745017901814010207

17. Lenk M, Berth H, Joraschky P, Petrowski K, Weidner K, Hannig C (2013) Fear of dental treatment-an underrecognized symptom in people with impaired mental health. Deutsches Arzteblatt international 110(31-32):517-522. https://doi.org/10.3238/arztebl.2013. 0517

18. Nociti FH Jr, Casati MZ (2000) Duarte PM (2015) Current perspective of the impact of smoking on the progression and treatment of periodontitis. Periodontol 67(1):187-210. https:// doi.org/10.1111/prd.12063

19. Finkelstein DM, Kubzansky LD, Goodman E (2006) Social status, stress, and adolescent smoking. J Adolesc Health 39(5):678-685. https://doi.org/10.1016/j.jadohealth.2006.04.011

20. Jané-Llopis E, Matytsina I (2006) Mental health and alcohol, drugs and tobacco: a review of the comorbidity between mental disorders and the use of alcohol, tobacco and illicit drugs. Drug Alcohol Rev 25(6):515-536. https://doi.org/10.1080/0959523060 0944461

21. Lasser K, Boyd JW, Woolhandler S, Himmelstein DU, McCormick D, Bor DH (2000) Smoking and mental illness: a populationbased prevalence study. JAMA 284(20):2606-2610. https://doi. org/10.1001/jama.284.20.2606

22. Smith PH, Mazure CM, McKee SA (2014) Smoking and mental illness in the U.S. population. Tob Control 23 (e2):e147-153. https://doi.org/10.1136/tobaccocontrol-2013-051466

23. Cooper J, Mancuso SG, Borland R, Slade T, Galletly C, Castle D (2012) Tobacco smoking among people living with a psychotic illness: the second Australian Survey of Psychosis. Aust N Z J Psychiatry 46(9):851-863. https://doi.org/10.1177/0004867412 449876

24. McKee SA, Falba T, O’Malley SS, Sindelar J, O’Connor PG (2007) Smoking status as a clinical indicator for alcohol misuse in US adults. Arch Intern Med 167(7):716-721. https://doi.org/ 10.1001/archinte.167.7.716

25. Wang J, Lv J, Wang W, Jiang X (2016) Alcohol consumption and risk of periodontitis: a meta-analysis. J Clin Periodontol 43(7):572-583. https://doi.org/10.1111/jcpe. 12556

26. Tonetti MS, Claffey N (2005) Advances in the progression of periodontitis and proposal of definitions of a periodontitis case and disease progression for use in risk factor research. Group C consensus report of the 5th European Workshop in Periodontology. J Clin Periodontol 32 Suppl 6:210-213. https://doi.org/10. 1111/j.1600-051X.2005.00822.x

27. Silness J, Loe H (1964) Periodontal disease in pregnancy. II. Correlation between oral hygiene and periodontal condition. Acta Odontol Scand 22:121-135

28. Loe H (1967) The gingival index, the plaque index and the retention index systems. J Periodontol 38 (6):Suppl:610-616. https:// doi.org/10.1902/jop.1967.38.6.610

29. Corah NL (1969) Development of a dental anxiety scale. J Dent Res 48(4):596. https://doi.org/10.1177/002203456904800 41801

30. Kroenke K, Strine TW, Spitzer RL, Williams JB, Berry JT, Mokdad AH (2009) The PHQ-8 as a measure of current depression in the general population. J Affect Disord 114(1-3):163-173. https:// doi.org/10.1016/j.jad.2008.06.026

31. Spitzer RL, Kroenke K, Williams JB, Löwe B (2006) A brief measure for assessing generalized anxiety disorder: the GAD-7. Arch Intern Med 166(10):1092-1097. https://doi.org/10.1001/ archinte.166.10.1092

32. Gierk B, Kohlmann S, Kroenke K, Spangenberg L, Zenger M, Brähler E, Löwe B (2014) The somatic symptom scale-8 (SSS-8): a brief measure of somatic symptom burden. JAMA Intern Med 174(3):399-407. https://doi.org/10.1001/jamainternmed.2013. 12179

33. Grabe HJ, Schulz A, Schmidt CO, Appel K, Driessen M, Wingenfeld K, Barnow S, Spitzer C, John U, Berger K, Wersching H, Freyberger HJ (2012) A brief instrument for the assessment of childhood abuse and neglect: the childhood trauma screener (CTS). Psychiatr Prax 39(3):109-115. https://doi.org/10.1055/s0031-1298984

34. Morgan JF, Reid F, Lacey JH (1999) The SCOFF questionnaire: assessment of a new screening tool for eating disorders. BMJ 
(Clinical research ed) 319(7223):1467-1468. https://doi.org/10. 1136/bmj.319.7223.1467

35. Babor TF, Higgins-Biddle J, Saunders J, Monteiro M (2001) AUDIT: the alcohol use disorders identification test: guidelines for use in primary health care. World Health Organization. https:// apps.who.int/iris/handle/10665/67205. Accessed 16 June 2021

36. Heatherton TF, Kozlowski LT, Frecker RC, Fagerström KO (1991) The Fagerström Test for Nicotine Dependence: a revision of the Fagerström Tolerance Questionnaire. Br J Addict 86(9):1119-1127. https://doi.org/10.1111/j.1360-0443.1991. tb01879.x

37. Bortz J, Schuster C (2011) Statistik für Human-und Sozialwissenschaftler: Limitierte Sonderausgabe. Springer-Verlag

38. Liu F, Wen YF, Zhou Y, Lei G, Guo QY, Dang YH (2018) A meta-analysis of emotional disorders as possible risk factors for chronic periodontitis. Medicine 97(28):e11434. https://doi.org/10. 1097/md.0000000000011434

39. Larijani HH, Guggisberg M (2015) Improving clinical practice: what dentists need to know about the association between dental fear and a history of sexual violence victimisation. Int J Dent 2015:452814. https://doi.org/10.1155/2015/452814

40. Leeners B, Stiller R, Block E, Görres G, Imthurn B, Rath W (2007) Consequences of childhood sexual abuse experiences on dental care. J Psychosom Res 62(5):581-588. https://doi.org/10. 1016/j.jpsychores.2006.11.009

41. Merikangas KR, He JP, Burstein M, Swanson SA, Avenevoli S, Cui L, Benjet C, Georgiades K, Swendsen J (2010) Lifetime prevalence of mental disorders in U.S. adolescents: results from the National Comorbidity Survey Replication--Adolescent Supplement (NCS-A). J Am Acad Child Adolesc Psychiatry 49 (10):980-989. https://doi.org/10.1016/j.jaac.2010.05.017

42. Locker D, Poulton R, Thomson WM (2001) Psychological disorders and dental anxiety in a young adult population. Commun Dent Oral Epidemiol 29(6):456-463. https://doi.org/10.1034/j. 1600-0528.2001.290607.x

43. Borrell LN (2000) Crawford ND (2012) Socioeconomic position indicators and periodontitis: examining the evidence. Periodontol 58(1):69-83. https://doi.org/10.1111/j.1600-0757.2011.00416.x

44. Boillot A, El Halabi B, Batty GD, Rangé H, Czernichow S, Bouchard P (2011) Education as a predictor of chronic periodontitis: a systematic review with meta-analysis population-based studies. PLoS ONE 6(7):e21508. https://doi.org/10.1371/journ al.pone. 0021508

45. Eke PI, Dye BA, Wei L, Slade GD, Thornton-Evans GO, Borgnakke WS, Taylor GW, Page RC, Beck JD, Genco RJ (2015) Update on prevalence of periodontitis in adults in the United States: NHANES 2009 to 2012. J Periodontol 86(5):611-622. https://doi.org/10.1902/jop.2015.140520

46. Coelho JMF, Miranda SS, da Cruz SS, Trindade SC, PassosSoares JS, Cerqueira EMM, Costa M, Figueiredo A, Hintz AM, Barreto ML, Seymour GJ, Scannapieco F, Gomes-Filho IS (2020) Is there association between stress and periodontitis? Clin Oral Invest 24(7):2285-2294. https://doi.org/10.1007/ s00784-019-03083-9

47. Stöhr J, Barbaresko J, Neuenschwander M, Schlesinger S (2021) Bidirectional association between periodontal disease and diabetes mellitus: a systematic review and meta-analysis of cohort studies. Sci Rep 11(1):13686. https://doi.org/10.1038/ s41598-021-93062-6

48. Woelber JP, Gärtner M, Breuninger L, Anderson A, König D, Hellwig E, Al-Ahmad A, Vach K, Dötsch A, Ratka-Krüger P, Tennert C (2019) The influence of an anti-inflammatory diet on gingivitis. A randomized controlled trial. J Clin Periodontol 46 (4):481-490. https://doi.org/10.1111/jcpe.13094

49. Guentsch A, Stier C, Raschke GF, Peisker A, Fahmy MD, Kuepper $\mathrm{H}$, Schueler I (2017) Oral health and dental anxiety in a German practice-based sample. Clin Oral Invest 21(5):1675-1680. https:// doi.org/10.1007/s00784-016-1951-8

50. Liu Y, Huang X, Yan Y, Lin H, Zhang J, Xuan D (2015) Dental fear and its possible relationship with periodontal status in Chinese adults: a preliminary study. BMC Oral Health 15:18. https:// doi.org/10.1186/1472-6831-15-18

51. Ng SK, Leung WK (2008) A community study on the relationship of dental anxiety with oral health status and oral health-related quality of life. Commun Dent Oral Epidemiol 36(4):347-356. https://doi.org/10.1111/j.1600-0528.2007.00412.x

52. Armfield JM, Slade GD, Spencer AJ (2009) Dental fear and adult oral health in Australia. Commun Dent Oral Epidemiol 37(3):220-230. https://doi.org/10.1111/j.1600-0528.2009.00468.x

53. Eitner S, Wichmann M, Paulsen A, Holst S (2006) Dental anxiety-an epidemiological study on its clinical correlation and effects on oral health. J Oral Rehabil 33(8):588-593. https://doi.org/10. 1111/j.1365-2842.2005.01589.x

54. Liu Y, Zhang C, Wu J, Yu H, Xie C (2021) Evaluation of the relationship among dental fear, scaling and root planing and periodontal status using periodontitis stages: a retrospective study. J Dent Sci. https://doi.org/10.1016/j.jds.2021.04.002

55. Enkling N, Jöhren H, Bürklein S, Lenk M, Margraf-Stiksrud J, Beck G, Daubländer M, Wölber J, Wannemüller A, Dünniger P (2019) S3-Leitlinie Zahnbehandlungsangst beim Erwachsenen (AWMF-Registernummer: 083-020).

56. Wannemueller A, Jöhren HP, Borgstädt A, Bosch J, Meyers M, Völse M, Scholten S, Margraf J (2016) Large group exposure treatment: a feasibility study of exposure combined with diaphragmatic breathing in highly dental fearful individuals. Front Psychol 7:2007. https://doi.org/10.3389/fpsyg.2016.02007

57. de Jongh A, Muris P, ter Horst G, van Zuuren F, Schoenmakers N, Makkes P (1995) One-session cognitive treatment of dental phobia: preparing dental phobics for treatment by restructuring negative cognitions. Behav Res Ther 33(8):947-954. https://doi. org/10.1016/0005-7967(95)00027-u

58. Maughan B, Rutter M (1997) Retrospective reporting of childhood adversity: issues in assessing long-term recall. J Pers Disord 11(1):19-33. https://doi.org/10.1521/pedi.1997.11.1.19

59. Lampert T, Kroll L, Müters S, Stolzenberg H (2013) Measurement of the socioeconomic status within the German Health Update 2009 (GEDA). Bundesgesundheitsblatt Gesundheitsforschung Gesundheitsschutz 56(1):131-143

Publisher's note Springer Nature remains neutral with regard to jurisdictional claims in published maps and institutional affiliations. 